\title{
Synchronization in simple network motifs with negligible correlation and mutual information measures
}

\author{
Miguel C. Soriano, ${ }^{1}$ Guy Van der Sande, ${ }^{2}$ Ingo Fischer, ${ }^{1}$ and Claudio R. Mirasso ${ }^{1}$ \\ ${ }^{1}$ Instituto de Física Interdisciplinar y Sistemas Complejos, IFISC (CSIC-UIB), \\ Campus Universitat Illes Balears, E-07122 Palma de Mallorca, Spain \\ ${ }^{2}$ Applied Physics Research Group (APHY), Vrije Universiteit Brussel, Pleinlaan 2, 1050 Brussels, Belgium
}

(Dated: January 31, 2012)

\begin{abstract}
Can different or even identical coupled oscillators be completely uncorrelated and still be synchronized? What can be concluded from the absence of correlations or even mutual information in networks of dynamical elements about their connectivity? These are fundamental and far-reaching questions arising in many complex systems. In this manuscript we address these two questions and demonstrate in simple and generic network motifs that synchronized behavior in the generalized sense can be realized and constructed such that no correlations and even negligible mutual information remain. Our findings raise new questions, in particular whether and to what extent indirect connections are being underestimated, since the related collective behavior and even synchronization is less likely to be detected.
\end{abstract}

PACS numbers: 05.45.Xt,05.45.Pq, 05.45.Jn, 05.45.Tp

The study of synchronization in coupled dynamical systems dates back to the works of Huygens in the 17th century [1]. When addressing synchronization in coupled complex dynamical systems, often the focus is on the particular case of an identically synchronized state [2]. However, synchronization in a generalized sense can still exist, even if there is no one-to-one relationship between the responses of the coupled systems [3]. Generalized synchronization requires a relationship between the dynamics of the constituting systems, no matter how complex this relationship is. If such a relationship exists, the response of one system is completely determined by the other one. The interaction between the systems can be either bidirectional or unidirectional. In the latter case, also known as drive-response configuration, the dynamics of the response system is, after transients, fully reproducible for the repetition of the drive signals, i.e. generalized synchronization boils down to the notion of consistency [4]. From an information theory perspective, synchronization requires a minimum amount of information to be transferred between the coupled elements. This minimum information has been determined precisely for the example of a system of coupled chaotic oscillators [5].

The dynamics of coupled systems has been extensively studied in biological networks [6, 7], lasers [8], neural networks [9], and many other self-organizing systems. One of the key ingredients in many of these studies is the network topology in which the dynamical elements are embedded. The behavior that emerges from the interaction strongly depends on the underlying network. From a theoretical point of view different topologies have been extensively considered and analyzed [10]. In real-world systems, however, the underlying network topology is often unknown and only measured time series of a subset of elements or of a mean field are available. Prominent examples are climate modeling [11], ecological modeling
[12] and neuroscience [13], among others. In all these areas correlation measures are being extensively used to deduce functional or, in some cases, effective connectivity. Functional connectivity assumes statistical dependencies between distinct units of the system while effective connectivity refers to causal interactions among the constituents. Functional connectivity, in turn, is used to develop models and to conclude on the abilities of a network [14].

The problem of network reconstruction is particularly important in neuroscience, where it has been identified that several diseases and impairments are related to changes in the network topology [15]. In human neurophysiology, mostly functional magnetic resonance (fMRI), electroencephalography (EEG) or magneto encephalography (MEG) data are available. These data boil down to a measurement of average activity of an already large ensemble of neurons. To unveil the connectivity information, several techniques have been used, particularly cross-correlation and mutual entropy are the most widely considered (see e.g. [13] and references therein). However, as we will show below, these two indicators can, under certain circumstances, underestimate and completely miss indirect connections. It is our aim to show in this Letter, via modeling and experiments, that for some simple configurations of coupled dynamical elements negligible correlation or mutual information are observed, although the elements are synchronized and determine each other's behaviors completely.

We have chosen different topologies, which can be classified into two general categories: bidirectional and unidirectional coupling schemes. Figure 1(a) illustrates a mutual interaction scheme while Fig. 1(b) depicts a driveresponse configuration. The square boxes in Fig. 1 account for different coupling interactions between dynamical elements $A$ and $B$. For illustration purposes, we 


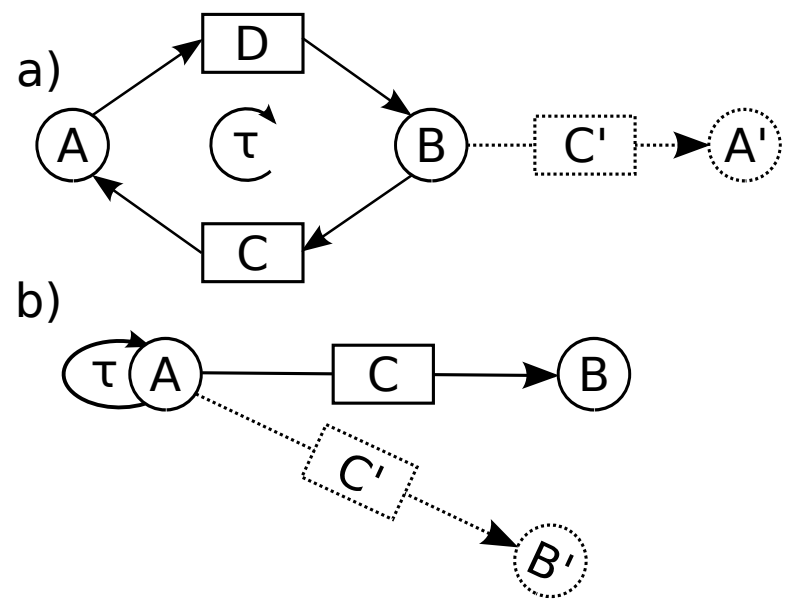

FIG. 1: Schematic drawing of two oscillators $(A$ and $B$ ) in (a) a mutual coupling arrangement with coupling paths $C$ and $D$, and (b) a drive-response configuration with a coupling path $C$. The dashed lines indicate the use of an auxiliary system approach to detect generalized synchronization. $A^{\prime}, B^{\prime}$, and $C^{\prime}$ are a copy of $A, B, C$, respectively.

have chosen Mackey-Glass oscillators (MGOs) $[16,17]$ as dynamical elements for all nodes in the network motifs. Nevertheless, our results can be extended to a larger class of nonlinear functions.

In the two topologies of Figure 1, we will first test the correlation properties between $A$ and $B$ for different number of nonlinear elements in the respective coupling paths. Subsequently, we will look for the existence of a generally synchronized state by testing the correlation properties of $A$ and $B$ with respect to auxiliary systems $A^{\prime}$ and $B^{\prime}$ (see Figure 1).

We start by studying the motif of two mutually coupled systems with delay as depicted in Fig. 1(a). We consider coupling paths, $C$ and $D$, that include a unidirectionally delay-coupled chain of oscillators each [18]. In this configuration, delay is included in the coupling path to generate oscillations, since the isolated MGO operates in a stable regime. In practice, the way the delay is distributed among the coupled elements is not relevant. The total delay in the coupling loop is the only relevant quantity, and can be located in a single path [18]. Previous studies show that this delay-coupled configuration does not exhibit identical synchronization of elements $A$ and $B$ when they operate in a chaotic regime [19-22]. Nevertheless, in-phase synchronization and rotating waves can eventually appear in the case of periodic dynamics [23].

We have investigated the dynamics generated in such a topology with an experimental implementation using MGOs (see [18, 24, 25] for electronic circuit implementation). In Figure 2 (a) and (b), we show the chaotic fluctuations of the individual MGOs (output voltages) $A$ and $B$, with coupling paths comprising 4 MGOs each (in $C$ and $D$ ), with a total delay time of $30 \mathrm{~ms}$ in the loop. The cross-correlation function [18] between elements $A$
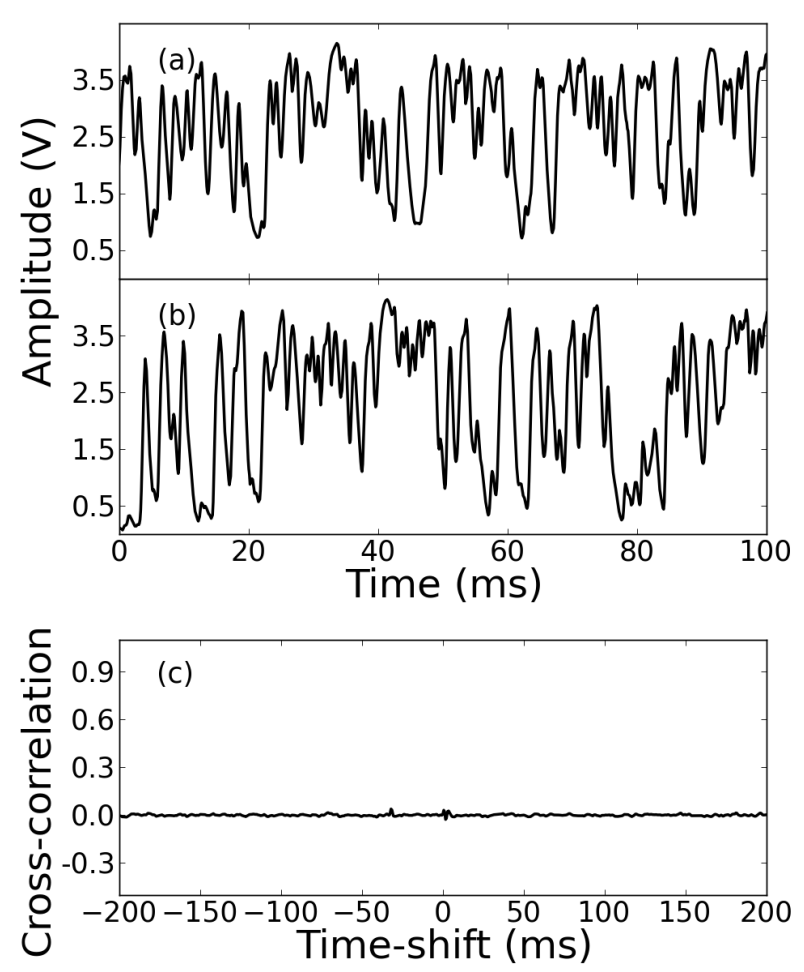

FIG. 2: Experimentally recorded time-trace of the chaotic dynamics of (a) element $A$ and (b) element $B$ in a mutual coupling configuration with $4 \mathrm{MGOs}$ in the coupling paths, with a delay time of $30 \mathrm{~ms}$. (c) Cross-correlation function between elements $A$ and $B$.

and $B$ is shown in Figure 2 (c). The maximum crosscorrelation (Xcorr) value between these two elements is 0.04. We have also computed the time delayed mutual information (DMI) [18] between them and found a maximum value of 0.05 . Additionally, we have performed experiments for a varying number $(N)$ of MGOs in the coupling paths. A summary of the experimental findings is presented in Figure 3 (a) and (b), solid lines. Both, the Xcorr and the DMI decay for an increasing number of MGOs in the coupling paths $C$ and $D$. The curve for the maxima of the cross-correlation shows an oscillating behavior, typical of the Mackey-Glass dynamics. The quantifiers have been estimated from time series of $2^{21}$ points.

The number of elements in the coupling paths is limited by the practical implementation. The values for Xcorr and DMI can be further lowered, if more elements are added in the coupling paths. We use a MGO model to extend our investigation to a larger number of elements. In the numerics, the dynamical equation describing the MGOs is an extension of the model for blood production proposed by Mackey and Glass [16], which has been extensively used in the characterization of chaotic systems [17]. The equation for the output of element $j$ in a coupled configuration reads

$$
\epsilon \dot{x}_{j}(t)=-x_{j}(t)+F\left[X_{j}(t)\right]
$$




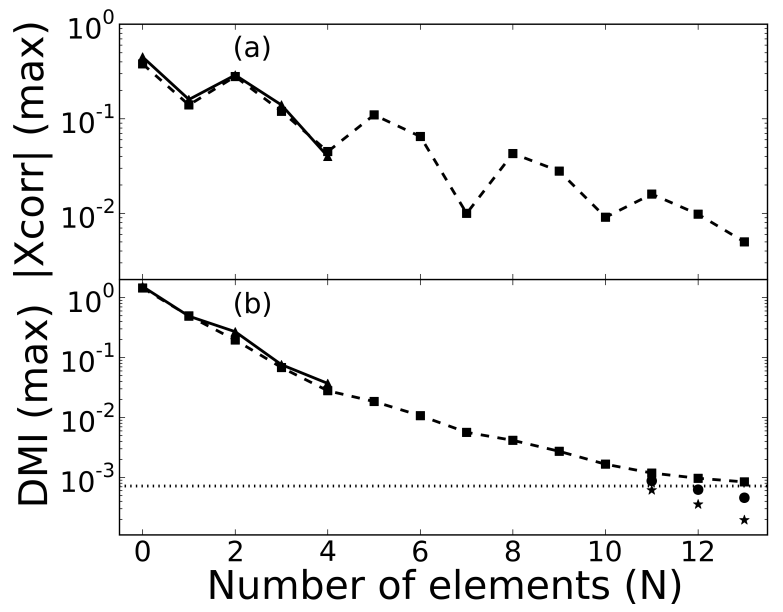

FIG. 3: Semi-log plot of the (a) absolute value of the crosscorrelation maxima and (b) mutual information maxima of elements $A$ and $B$ as a function of the number of elements in the coupling path $C$ (equal number in $D$ ) in the mutual coupling configuration, see Fig. 1(a). Dashed lines stand for the numerical results and solid lines for the experimental ones. Dotted line corresponds to the statistical noise floor due to the finite number of recorded points $\left(2^{21}\right)$. Circles correspond to the analysis of $2^{23}$ points and stars to $2^{25}$ points.

where $F\left[X_{j}\right]=a X_{j} /\left(1+b^{c} X_{j}^{c}\right)$. For the system depicted in Fig. $1(\mathrm{a}), X_{j}(t)=x_{j-1}\left(t-\frac{\tau}{(2 N+2)}\right)$, with $x_{j-1}$ being the element preceding $x_{j}$, and $N$ is the number of elements in each coupling path. The total delay in the ring, $\tau$, is $30 \mathrm{~ms}$ [18]. The argument of the nonlinear function $X_{j}$ changes for the other coupling topologies. The parameter values of the Mackey-Glass model are extracted from the fit of the experimentally recorded nonlinearity, yielding $\langle a\rangle=2.1,\langle b\rangle=1 / 3,\langle c\rangle=9.9$. The time constant of the experimental implementation, $\epsilon$, is 0.47 ms. The parameter mismatch found in the experimental implementation of the MGOs has been accounted for in the numerical simulations. The system dynamics is obtained by numerically integrating Eq. 1 using the Heun method with an integration time step of $0.01 \mathrm{~ms}$. We sample points at $0.1 \mathrm{~ms}$ for the correlation and mutual information analysis.

A summary of the numerical findings is presented in Figure 3 (a) and (b), dashed lines. It can be seen that the tendency towards smaller values of Xcorr and DMI continues when more elements are added in the coupling paths. For $0 \leq N \leq 4$, there is a perfect agreement between experimental and numerical results. For $N>$ 11, the numerical results of the DMI seem to saturate as they reach the statistical significance level for time series of $2^{21}$ points (dotted line), shown in Figure 3(b). An analysis for even longer time series yields values of DMI that continue decreasing, as shown by the circles and stars in Fig. 3(b).

In addition to the motif of two mutually coupled systems, we have further analyzed the simple drive-response

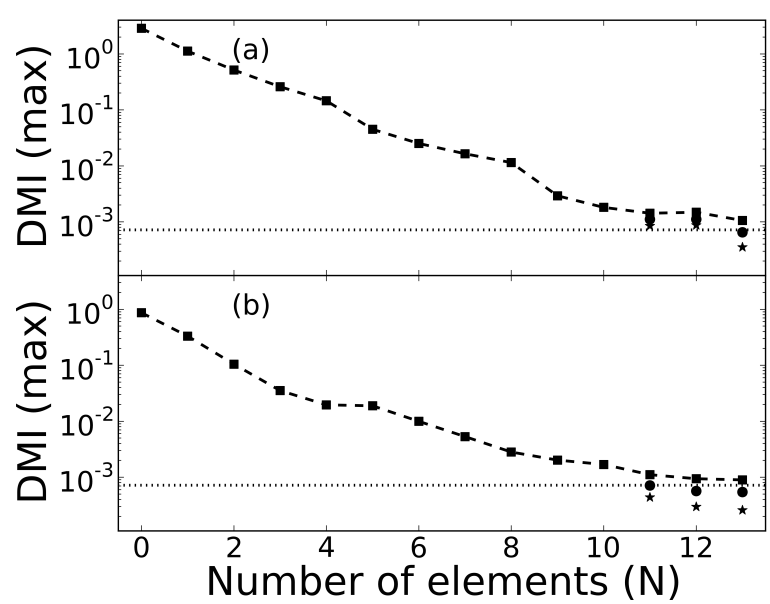

FIG. 4: Numerical results of the maxima of the mutual information shared by elements $A$ and $B$ for an increasing number of elements in the coupling path $C$ in the drive-response configuration, see Fig. 1(b). Element $B$ and MGOs in the coupling path have, in panel (a), coupling from the preceding element and, in panel (b), self-feedback and coupling from preceding element. Dotted line corresponds to the statistical noise floor due to the finite number of recorded points $\left(2^{21}\right)$. Circles for the analysis of $2^{23}$ points and stars to $2^{25}$ points.

motif, as depicted in Fig. 1(b). We inject a chaotic signal generated by a MGO with self-feedback $A$ into $B$ via $C$. The coupling path $C$ includes a chain of oscillators, which have parameter mismatch among them (diversity) to prevent identical synchronization to occur between neighbors (Gaussian distribution with variance 0.2 around parameters values). In this situation, we find that the correlation and mutual information between elements $A$ and $B$ decrease for an increasing number of MGOs in the coupling path (simulations are started at different initial conditions). In Figure 4 we show the results for the DMI for two different scenarios of the unidirectional coupling, namely element $B$ and oscillators in the coupling path with or without self-feedback [18]. We make this distinction since the MGOs without selffeedback are damped oscillators in the absence of coupling, while the MGOs with self-feedback can operate in a pulsating regime in the absence of coupling. First, we consider MGOs without self-feedback in $B$ and the coupling chain, i.e. $X_{j}(t)=x_{j-1}(t)$ in Eq. 1. In Fig. 4(a), it can be seen that the DMI also decays with an increasing number of elements in the coupling path. Second, we consider MGOs with self-feedback in $B$ and the coupling chain, i.e. $X_{j}(t)=x_{j-1}(t)+x_{j}(t-\tau)$ in Eq. 1. That is, the oscillators in the coupling path are subject to a self-feedback loop and are also coupled to the preceding element in the path. It is clear from Fig. 4(b) that the DMI decays with the number of elements in this configuration. This DMI decay is similar to the results for the mutually coupled topology in Fig. 3(b).

The neglibible amount of correlation and mutual in- 


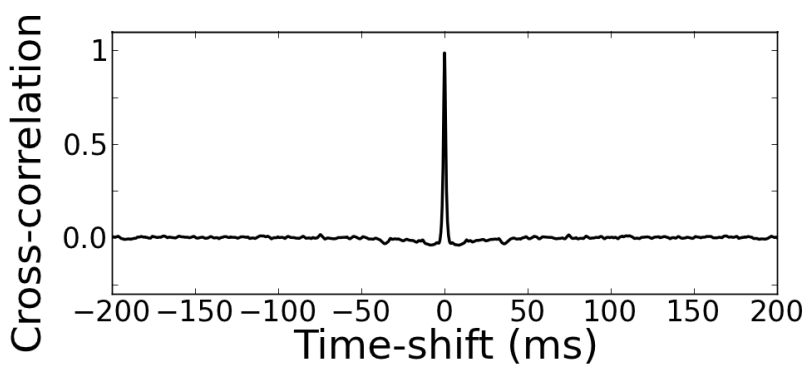

FIG. 5: Cross-correlation function between elements $A$ and $A^{\prime}$ in a mutual coupling configuration with 4 MGOs in the coupling paths.

formation measures between elements $A$ and $B$ raises the natural question on whether these elements might still be synchronized or not. The auxiliary system approach, proposed by Abarbanel and co-workers [26], is a practical test to check the existence of generalized synchronization. The auxiliary system approach requires the use of an exact replica of the response system, which is driven by exactly the same input as the response system, although starting from different initial conditions. By observing a stable regime of identical oscillations in the auxiliary and response systems, the dynamics of the drive and response systems are considered to be generally synchronized. In how far the auxiliary system approach corresponds to the precise definition of generalized synchronization is still under debate. For the two topologies in Figure 1, the first part of the auxiliary systems is a precise copy of the coupling paths between $A$ and $B$, i.e. $C^{\prime} \equiv C$. The detection of generalized synchronization requires the last element to be a copy of $A\left(A^{\prime} \equiv A\right)$ for the bidirectional scheme and a copy of $B\left(B^{\prime} \equiv B\right)$ for the drive-response configuration. In the following we check, first, the correlation between $A$ and $A^{\prime}$ in the mutual coupling scheme and, second, the correlation between $B$ and $B^{\prime}$ in the drive-response one.

The correlation function between $A$ and $A^{\prime}$ obtained in the experimental implementation of the mutually coupled MGOs is presented in Figure 5. Our experimental results show that an identically synchronized state indeed exists and is robust against electrical noise and some degree of diversity of our electronic components. We experimentally find a correlation of 0.99 between elements $A$ and $A^{\prime}$, as shown in Fig. 5. This proves a stable identical synchronization between the elements. For such a high correlation between elements $A$ and $A^{\prime}$ in the presence of parameter mismatch of the oscillators, elements in the coupling paths $C$ and $C^{\prime}$ can be different but they must match pair wise. The identical synchronization between elements $A$ and $A^{\prime}$ is mediated by element $B$, which has a very low correlation and share a very small mutual information with the synchronized oscillators. The existence of this identically synchronized state between $A$ and $A^{\prime}$ requires the existence of generalized synchronization be-

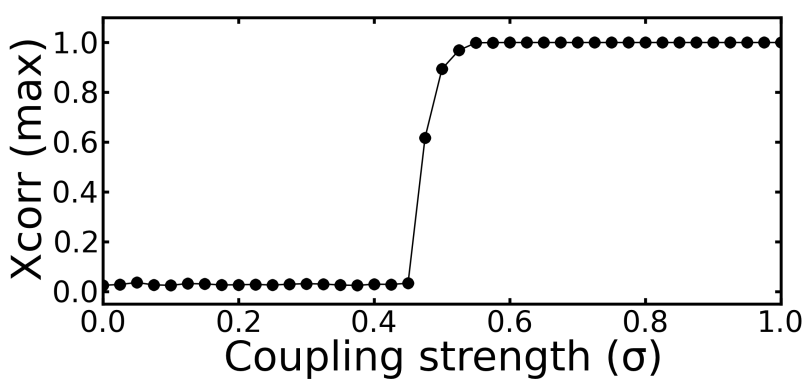

FIG. 6: Cross-correlation maxima between elements $B$ and $B^{\prime}$ in a drive-response configuration as a function of the normalized coupling strength $(\sigma)$. Normalized self-feedback strength $(1-\sigma) .10$ MGOs in the coupling paths $C$ and $C^{\prime}$.

tween $A$ and $B$. Interestingly, the amount of transferred information does not seem to be properly captured in our case by the here applied measures.

Additional numerical results have been performed in the mutual coupling and the drive-response configurations to support our findings. We find that the dynamical evolution of, respectively, elements $A-A^{\prime}$ and $B$ $B^{\prime}$ reach an identically synchronized state. This state appears after a transient time because simulations are started at different initial conditions proving the stability of the synchronized state. For the drive-response configuration, the scheme containing MGOs with self-feedback reaches the identically synchronized state provided that the coupling strength is larger than the self-feedback strength. Figure 6 shows the correlation between $B$ and $B^{\prime}$ as a function of the normalized coupling strength $(\sigma)$, when $X_{j}(t)=\sigma x_{j-1}(t)+(1-\sigma) x_{j}(t-\tau)$ in Eq. 1. For $\sigma<0.5$, the identically synchronized state is not reached. However, for $\sigma>0.5$, the synchronized state is reached with high correlation. Clearly, the limit $\sigma=1$ relates back to the oscillators without self-feedback.

In conclusion, we have shown both numerically and experimentally that under certain circumstances two elements that are connected via an uncorrelated signal can be generally synchronized. Our results are not restricted to Mackey-Glass oscillators, but are expected to be valid for any oscillator operating in the regime of consistency. It is shown that correlations and mutual information below the statistical limit, imposed by the finite number of data points, do not prevent identical synchronization to occur between distant elements. Our results highlight the extreme characteristics generalized synchronization can exhibit. Consequently, indirect connections might be underestimated when these techniques are applied to identify connections between distant areas in complex networks of dynamical elements. Since our presented network motifs are very generic, our results might apply to any networks with strong nonlinearities. This poses a strong challenge for the characterization of complex networks.

This work was supported by the MICINN (Spain), 
FEDER, and European Commission under Projects TEC2009-14101 (DeCoDicA), FIS2007-60237 (FISICOS), 0200950I190 (Proyecto Intramurales Especiales), and EC FP7 Project PHOCUS under Grant No. 240763.

[1] Ch. Huygens (Hugenii), Horologium Oscillatorium (Apud F. Muguet, Regis et Archiepiscopi Typographum, Paris, France, 1673), English translation: The Pendulum Clock (Iowa State University Press, Ames, 1986).

[2] H. Fujisaka and T. Yamada, Prog. Theor. Phys. 69, 32 (1983).

[3] L.M. Pecora, T.L. Carroll, G.A. Johnson, D.J. Mar, and J.F. Heagy, Chaos 7, 520 (1997).

[4] A. Uchida, R. McAllister, and R. Roy, Phys. Rev. Lett. 93, 244102 (2004).

[5] S. D. Pethel, N. J. Corron, Q. R. Underwood, and K. Myneni, Phys. Rev. Lett. 90, 254101 (2003).

[6] S.H. Strogatz and I. Stewart, Sci. Am. 269, 102 (1993).

[7] J. Garcia-Ojalvo, M.B. Elowitz, and S.H. Strogatz, Proc. Nat. Acad. Sci. USA 101, 10955 (2004).

[8] H.G. Winful and L. Rahman, Phys. Rev. Lett. 65, 1575 (1990).

[9] J.J. Hopfield, Proc. Nat. Acad. Sci. USA 79, 2554 (1982).

[10] S. Boccaletti, V. Latora, Y. Moreno, M. Chavez, and D.U. Hwang, Phys. Rep. 424, 175 (2006).

[11] J.F. Donges, Y. Zou, N. Marwan, and J. Kurths, Eur.
Phys. J. Special Topics 174, 157 (2009).

[12] C.W. Osenberg, O. Sarnelle, S.D. Cooper, and R.D. Holt, Ecologoy 80, 1105 (1999).

[13] F. Varela, J.P. Lachaux, E. Rodriguez, and J. Martinerie, Nat. Rev. Neurosci. 2, 229 (2001).

[14] C. Zhou, L. Zemanova, G. Zamora, C.C. Hilgetag, and J. Kurths, Phys. Rev. Lett. 97, 238103 (2006).

[15] C.J. Stam et al., Brain 132, 213 (2009).

[16] M.C. Mackey and L. Glass, Science 197, 287 (1977).

[17] J.D. Farmer, Physica D 4, 366 (1982).

[18] See Supplementary Material.

[19] T. Heil, I. Fischer, W. Elsasser, J. Mulet, and C.R. Mirasso, Phys. Rev. Lett. 86, 795 (2001).

[20] G. Van der Sande, M.C. Soriano, I. Fischer, and C.R. Mirasso, Phys. Rev. E 77, 055202(R) (2008).

[21] P. Perlikowski, S. Yanchuk, M. Wolfrum, A. Stefanski, P. Mosiolek, and T. Kapitaniak, Chaos 20, 013111 (2010).

[22] D. V. Senthilkumar, P. Muruganandam, M. Lakshmanan, and J. Kurths, Phys. Rev. E 81, 066219 (2010).

[23] P. Perlikowski, S. Yanchuk, O. V. Popovych, and P. A. Tass, Phys. Rev. E. 82, 036208 (2010).

[24] A. Namajunas, K. Pyragas, and A. Tamasevicius, Phys. Lett. A 201, 42 (1995).

[25] S. Sano, A. Uchida, S. Yoshimori, and R. Roy, Phys. Rev. E 75, 016207 (2007).

[26] H.D.I. Abarbanel, N.F. Rulkov, and M.M. Sushchik, Phys. Rev. E 53, 4528 (1996). 